\title{
Téoros
}

Revue de recherche en tourisme

\section{Cette route a-t-elle un nom ou une histoire ?}

\section{Martin Drouin}

Volume 25, numéro 3, automne 2006

URI : https://id.erudit.org/iderudit/1071023ar

DOI : https://doi.org/10.7202/1071023ar

Aller au sommaire du numéro

Éditeur(s)

Université du Québec à Montréal

ISSN

0712-8657 (imprimé)

1923-2705 (numérique)

Découvrir la revue

Citer cette note

Drouin, M. (2006). Cette route a-t-elle un nom ou une histoire ? Téoros, 25(3),

73-76. https://doi.org/10.7202/1071023ar d'utilisation que vous pouvez consulter en ligne.

https://apropos.erudit.org/fr/usagers/politique-dutilisation/ 


\section{Cette route a-t-elle un nom
Cemaire de recherche du Canada en patrimoine ou une histoire?}

\section{Martin Drouin}

En route pour la baie Sainte-Marie en Nouvelle-Écosse, mon attention fut attirée par un panneau routier annonçant : Evangeline Trail. Le chemin que j'empruntais n'avait pas seulement le numéro d'identification habituel, mais il portait aussi un véritable nom. L'héroïne du poète Henry Wadsworth Longfellow m'indiquait que j'entrais dans I'un des foyers acadiens des provinces maritimes. La foule d'objets hétéroclites aux couleurs du drapeau national, exposés sur les devantures de maison, enlevait tout doute quant à l'appartenance de la population locale. La route à thème avait donc misé sur le caractère identitaire du lieu : le personnage légendaire servait de principe organisateur pour faire découvrir aux touristes ce coin de pays. J'étais en revanche perplexe devant le texte de la brochure touristique qui m'invitait à découvrir « le pays d'Évangéline ". J'y trouvais en effet les formules habituelles qui m'annonçaient un «voyage riche en culture, en diversité, en charme et en beauté naturelle ». La justification du choix du thème n'était guère concluante. Une phrase expéditive à la fin d'un paragraphe évoquait tout juste le nom et l'auteur du poème. Le traitement d'un épisode historique douloureux avait-il fait peur aux experts en commercialisation pour qu'ils s'en tiennent à des formules aussi creuses? Les quelques jours à sillonner les routes de la région et la visite du lieu historique national de Grand-Pré, "foyer historique le plus important du peuple acadien " ${ }^{1}$, avaient pourtant amplement fourni matière à interprétation.

L'objectif de la route touristique avait tout de même été réalisé. Le thème avait réussi à souligner la présence d'une communauté, à marquer de son sceau l'invisible; la curiosité me poussait même à lire les longs vers du poème épique. Ainsi appelée à jouer de ses charmes, l'histoire était parvenue à me tenir en éveil et à animer les discussions dans la voiture. Vous le devinez, le terreau d'historien était bien sûr fertile pour une telle impression favorable! J'allais cependant être un peu déçu, sur la portion sud de la province, où l'Evangeline Trail s'était transformée en Lighthouse Route. Cette fois, la brochure impressionnait en lyrisme pour présenter " la route des phares". Ce n'est pas tellement les promesses de croiser des « attelages de bœufs tirer des charges de bois", de voir des "pêcheurs prenant le large à la rame " ou d'entendre le " forgeron marteler encore le fer sur son enclume au milieu des immeubles magnifiquement préservés du quartier historique " qui avait frustré mes attentes; le pittoresque se mêlait décidément à une vision romantique du passé pour vendre la région. J'étais plutôt surpris - et ma réaction était peut-être naiive du traitement sans nuance des deux thèmes rencontrés. Dans mon esprit, l'histoire d'Évangéline et les phares ne pouvaient pas béné-

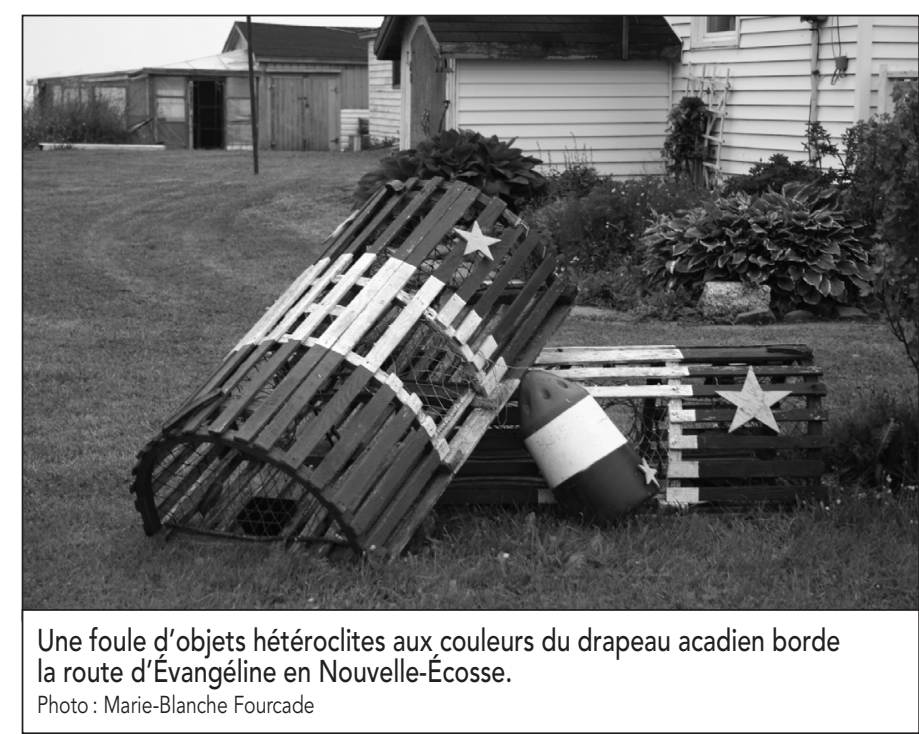

ficier d'une présentation similaire et être seulement différenciés par deux pictogrammes distincts. Mon étonnement s'accentua à l'entrée de l'île du Cap-Breton. À Port Hasting, un immense panneau routier stylisait le territoire pour répertorier les cinq routes thématiques offertes. En fait, j'allais m'en rendre compte plus tard, l'ensemble des voies côtières de la province avait été ainsi baptisé ${ }^{2}$. En tout, 11 routes thématiques quadrillaient la Nouvelle-Écosse. La pratique était bel et bien implantée.

Le Québec n'échappe guère à la tendance. Depuis 2000, huit routes officiellement identifiées serpentent majoritairement la vallée du Saint-Laurent. L'instigateur du projet, Tourisme Québec, avance que celles-ci « améliore[nt] l'expérience touristique des visiteurs » parce qu'elles « mettent en valeur le caractère distinctif d'une région et [...] font découvrir des paysages souvent exceptionnels ainsi que des attraits ou des activités touristiques qui valent le détour " ${ }^{3}$. La signalisation, inscrite dans un plan stratégique mis en place depuis les années 1980, guide intelligemment l'automobiliste et l'aide à satisfaire sa curiosité. II est d'ailleurs intéressant de remarquer que, d'un système signalétique défini en termes de lieux ou de sites, c'est-àdire en identifiant des attraits, des activités et des hébergements, la tendance récente s'est étendue à penser des parcours en dehors des grands axes routiers communément empruntés. Le geste contribue à un mouvement plus général que les revues Téoros et Continuité, par exemple, ont présenté dans des numéros thématiques au cours de l'été 2003. La publication en tourisme se pen- 
chait alors sur les « routes à thèmes », tandis que le périodique du Conseil des monuments et sites du Québec, dans un dossier consacré au tourisme culturel, s'intéressait au " patrimoine en circuits ». La recension des expériences alors implantées pour l'ensemble de la province permettait facilement de conclure que les milieux patrimoniaux étaient semblablement touchés par une fièvre de mise en circuit. Le tourisme et le patrimoine participaient ainsi à un même effort qui associait l'automobile.

Pourtant, lorsqu'il est question de patrimoine, l'automobile est souvent évoquée à propos des méfaits qu'elle engendre. Les détracteurs abondent pour l'accuser de bouleverser les paysages avec les kilomètres et les kilomètres d'asphalte qu'elle exige sous son passage, sans parler des inévitables destructions du cadre bâti. Elle serait aussi responsable de l'étalement urbain et, conséquence directe, de l'augmentation de mètres carrés de stationnement dans les centres-villes historiques. L'automobile fut même pendant un temps regardée comme l'un des ennemis publics de la conservation du patrimoine. La loi de l'île d'Orléans,

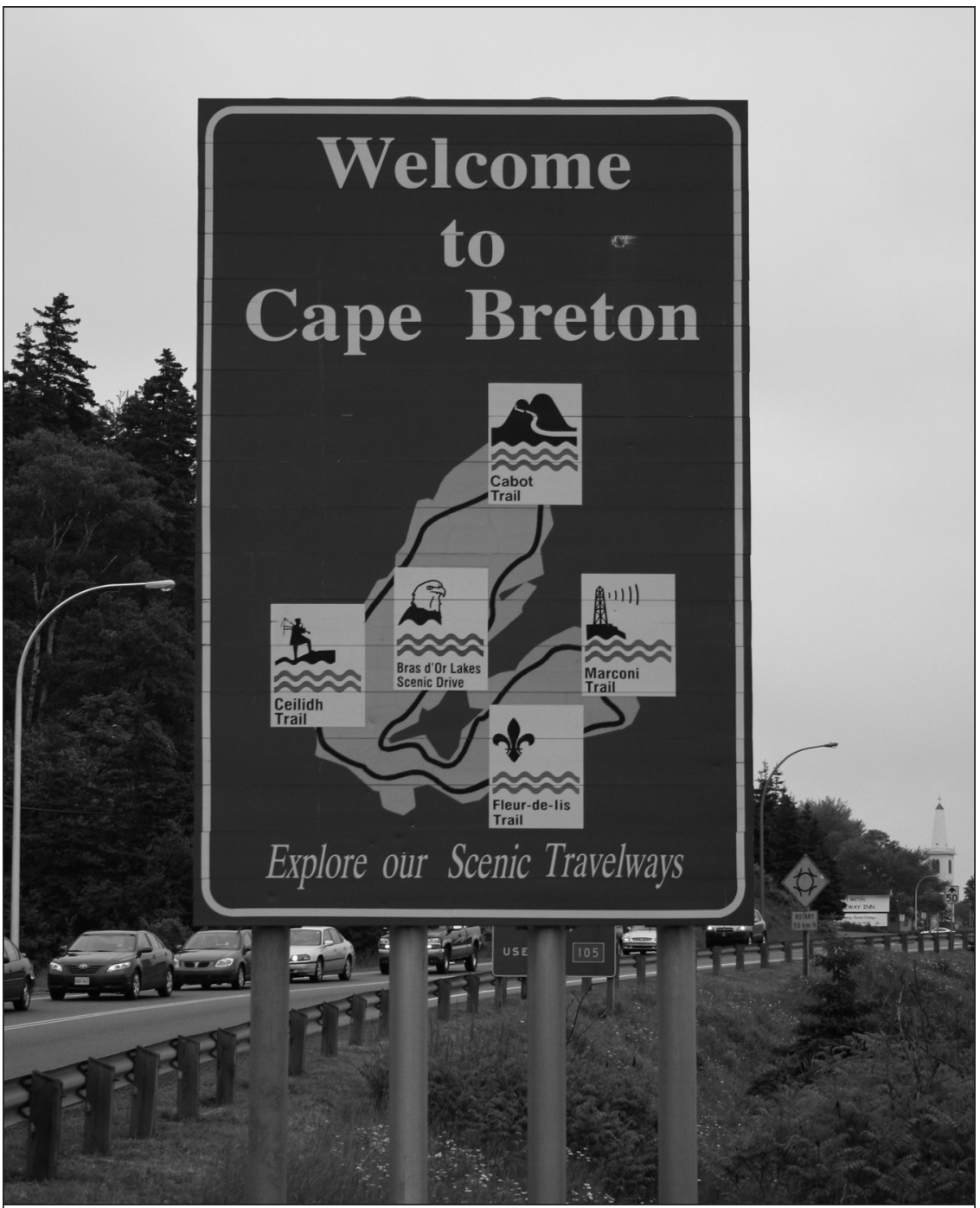

À Port Hasting, un panneau routier indique la présence de cinq routes touristiques sur l'île du Cap-Breton.

Photo: Jonathan Cha adoptée en 1935, n'était-elle pas la résultante de la construction du pont et de l'arrivée prévisible de la voiture motorisée ? Le projet visait de la sorte à préserver de la modernité le berceau des francophones en Amérique (De Blois Martin, 1997). La longue liste de récriminations n'est pas, somme toute, complètement injustifiée. II est pourtant paradoxalement facile d'affirmer, et la récente vogue des circuits l'exprime éloquemment, que l'automobile constitue un fabuleux moyen de découvrir le patrimoine.

Les avantages de l'automobile dans l'expérience touristique du patrimoine sont effectivement nombreux. En voyage, aucun horaire fixe ne vient bousculer l'heure du départ ou presser la visite d'un site pour reprendre l'autocar ou ne pas rater le train. Derrière un volant, une localisation excentrée redevient accessible. II serait en effet difficile d'imaginer atteindre l'anse au Meadows, à Terre-Neuve, ou le Canada York Factory, au Manitoba, sans un tel véhicule. À l'encontre d'autres moyens de locomotion, aucun parcours déterminé ne bride le désir d'errer ou de vagabonder ; l'itinéraire reste à l'entière discrétion du voyageur, ce que les circuits viennent en quelque sorte freiner. Le trajet, de plus, ne se pense pas point par point, c'est-à-dire de lieux en lieux comme le nécessite l'usage des gares ou des aéroports, mais selon un fil continu. Bien sûr, il s'agit toujours de se rendre à un endroit, à la différence que la distance parcourue peut devenir aussi importante que l'atteinte de l'objectif. L'union entre l'automobile, le tourisme et le patrimoine, c'est-à-dire de l'arrivée des circuits patrimoniaux, faut-il le rappeler, n'est pas si récente. Le tour de la Gaspésie, par exemple, aujourd'hui devenu un classique du genre, correspond à la construction de la première route dans les années 1930 (Gagnon, 2003 : 264-273). Une trentaine d'années plus tard, la reconstruction de la forteresse de Louisbourg servait de moteur économique pour revitaliser l'industrie touristique d'une région excentrée de la Nouvelle-Écosse (MacLean, 1995). L'ouverture du parc historique de la Pointe-du-Moulin dans les années 1970, à l'extrémité du boulevard Don-Quichotte à l'île Perrot, était semblablement pensée. II faut se rendre à l'évidence : patrimoine et automobile peuvent aussi faire bon ménage.

Les routes patrimoniales semblent exploiter à merveille cette union. Elles invitent en quelque sorte le touriste à se laisser guider à travers une expérience d'interprétation muséale à grande échelle. Le thème, clé de lecture du paysage, révèle les particularités et l'essence d'une région. Davantage que la simple succession d'attraits ou l'exploitation d'un site unique faisant office de produit d'appel, le parcours propose un récit à l'aide d'une unité spatiale qui favorise la compréhension de l'ensemble plutôt que de la somme de ses parties. À cet égard, l'Evangeline Trail, tout en étant minimale dans l'interprétation, donne à saisir l'esprit du lieu, celui du "grand dérangement " et du ré-enracinement des Acadiens, qui se superpose ainsi à la route physique. La signalisation aide le voyageur le plus paresseux à ouvrir les yeux. Libre à lui de 
se lancer dans une démarche approfondie pour en comprendre davantage. Reste à savoir s'il le pourra, car l'information offerte est en effet plutôt mince pour qui veut aller plus loin. Je l'évoquais précédemment à propos de la "route d'Évangéline ", une visite sur le site Internet officiel du gouvernement du Québec suffit pour finir de s'en convaincre. Au-delà de la garantie de "découvrir le Québec dans toute son authenticité " à travers "les trésors, culturels et naturels » des régions qu'elle traverse, il n'y a guère plus que quelques indications. Ainsi, le danger de la vogue des routes à thème n'est pas tellement leur multiplication qui risquerait de les banaliser, mais plutôt l'effet de mode qui, derrière les promesses de la thématisation, n'offre pas plus qu'un simple titre. Serait-il possible d'aller plus loin?

Les routes à thèmes n'oublient-elles pas l'essentiel ? Si le visiteur peut fréquenter l'histoire au cours du trajet, qu'advient-il de l'espace tangible sur lequel il circule ? Cet autre versant de l'objet a aussi attiré des spécialistes du patrimoine. II faut en effet distinguer les circuits patrimoniaux des routes historiques, c'est-à-dire celles qui mènent à des sites anciens, mais dont le tracé est relativement récent, de celles qui ont un passé qui les constitue. Différentes démarches ont vu le jour pour les mettre en valeur. Aux États-Unis, pays par excellence de la voiture, une association - Historic Roads - est même dédiée à l'identification, à la conservation et à la gestion des routes historiques (Historic Roads, 2004). Le registre du National Park Service protège d'ailleurs officiellement quelques-uns de ces chemins, dont l'un des plus célèbres la Route 66 est aujourd'hui une figure emblématique. Dans le même registre de protection étatique, le lieu historique national de la piste Chilkoot, au Yukon, est mis en valeur par Parcs Canada. Tourisme Yukon invite les randonneurs à emprunter la voie des premiers chercheurs d'or à la fin du XIXe siècle dans un « véritable musée vivant » (Tourisme Yukon, 2006). L'initiative rejoint l'injonction de certains spécialistes. En effet, à l'espace physique protégé s'ajouterait un caractère intangible lié à la fréquentation pérenne du site. Bref, en suivant les traces des premiers pionniers, le visiteur est invité à vivre une expérience similaire à celle vécue auparavant, sur le même chemin. Ainsi conçue, la route à thème est plutôt appelée un «itinéraire culturel » qui serait en quelque sorte " une vraie synthèse des valeurs matérielles et immatérielles » (Krestev, 2003) du patrimoine. II n'y a qu'un pas pour pouvoir définir une véritable offre de tourisme culturel.

Le récent succès des routes de pèlerinages le démontre éloquemment. La relance du Tro-Breiz en 1994, c'est-à-dire du tour historique de la Bretagne en l'honneur des sept saints fondateurs, répondait à une démarche spirituelle ; la réussite estivale non démentie des 12 dernières années rassemble désormais des randonneurs, d'un plus large horizon, venus vivre un temps hors de l'ordinaire. L'effervescence autour des chemins de Saint-Jacquesde-Compostelle exprime le même engouement et un processus de patrimonialisation semblable (Bertrand, 2005 : 26-38). Dans les années 1980, le Conseil de l'Europe s'est intéressé à valoriser et à traduire l'importance des anciennes routes de pèlerinage (Institut européen des itinéraires culturels, 2006) ${ }^{4}$. Au cours de la décennie suivante, l'Organisation des Nations unies pour l'éducation, la science et la culture (UNESCO) inscrivait d'abord sur la liste du patrimoine de l'humanité le segment espagnol du chemin de Compostelle, puis, cinq ans plus tard, la portion française du tracé. Ce fut d'ailleurs du-

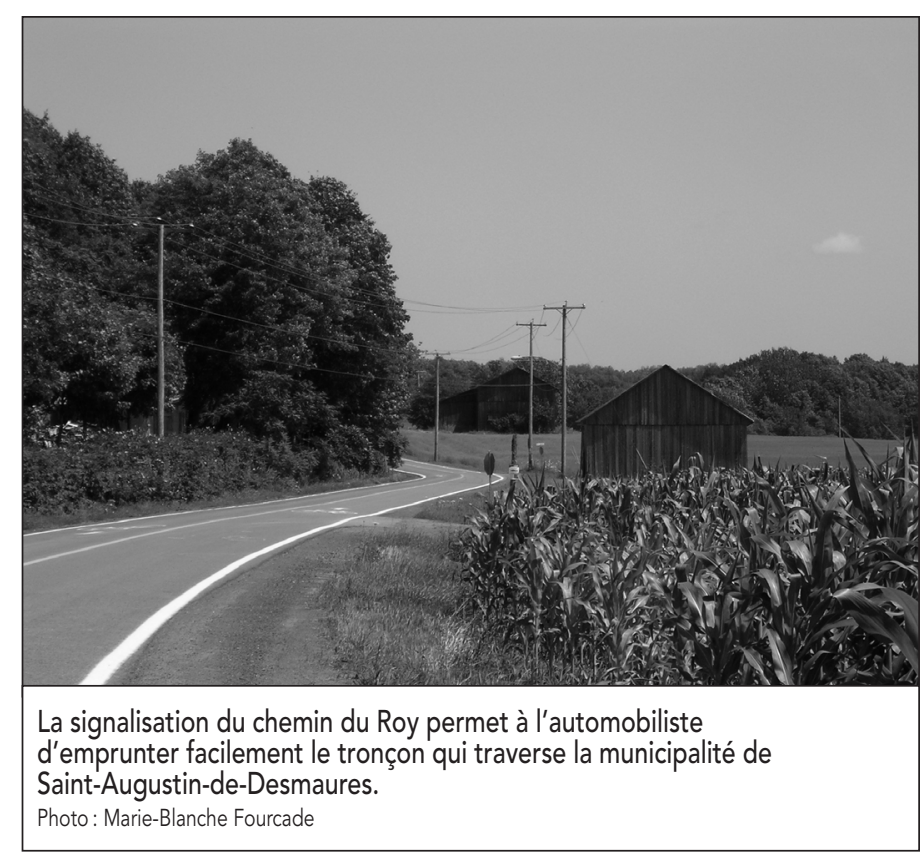

rant cette décennie que le Comité international des itinéraires culturels était formé au sein du Conseil international des monuments et des sites (ICOMOS) (Comité international des itinéraires culturels, 1994) ${ }^{5}$. En s'appuyant sur le texte de la Convention du patrimoine mondial, le groupe de scientifiques espérait trouver une façon de valoriser "la série en tant que telle et non ses éléments constitutifs pris individuellement " (Comité international des itinéraires culturels, 1998). Le terme de «paysage culturel linéaire » tentait de mettre un nom sur un patrimoine difficilement qualifiable (ICOMOS, 1998). Une volonté de partage interculturel, qui prendrait la forme d'un tourisme culturel à la même enseigne que les Intercultural Roads of Dialogue Projects menés par I'UNESCO ${ }^{6}$, anime les initiatives évoquées.

Par ailleurs, nombre de régions tentent par divers moyens d'attirer les touristes. Depuis 2000, le projet de valorisation des chemins de Craig et de Gosford espère, dans le cadre de différentes activités, faire reconnaître «l'unicité " et le " caractère historique » des deux routes (Circuit de découverte des chemins de Craig et Gosford, 2006). L'ancien parcours de Québec à Richmond, construit au début du XIXe siècle, a été balisé par une signalisation spécifique, mais aussi par l'aménagement d'infrastructures (aires de repos, haltes et belvédères), l'installation de panneaux d'interprétation, la restauration de bâtiments historiques et la mise en ligne d'un site Internet. Les moyens demeurent assez classiques, mais démontrent tout de même une certaine volonté. En 1996, Tourisme Québec choisissait de mettre en valeur le prestigieux chemin du Roy pour son projet pilote de routes touristiques. L'initiative a permis de redécouvrir, par exemple, une portion de son tracé difficilement identifiable dans la région de SaintAugustin-de-Desmaures, près de Québec. Une initiative de Lanaudière, dans le cadre du programme de collections numérisées du Canada, propose une exposition virtuelle d'une belle qualité sur le tronçon qui traverse la municipalité (Connexion-Lanaudière, 2003). L'itinéraire de Québec à Montréal avait déjà fait l'objet d'une publication de la Commission de toponymie au début des années 1980 (Bouchard, 1981). Connaissons-nous pour autant l'une des plus anciennes routes du Québec? 
"Cette route a-t-elle un nom ou une histoire? " devrait immanquablement se demander le voyageur au volant de sa voiture depuis le récent engouement des spécialistes en patrimoine et des experts en tourisme. Serait-il cependant possible de procéder à la synthèse entre les deux mondes ? L'espoir de transformer un périple en un espace d'apprentissage culturel et initiatique se communique-t-il autrement que par le biais d'expositions dans les institutions muséales ou par la virtualité du Web ? Où se trouve la route empruntée par le touriste à partir de ce moment? Les initiatives sont-elles dès lors seulement conçues comme un appel à se déplacer? À l'inverse, la signalisation qui annonce à l'automobiliste l'existence d'une route à thème marque-t-elle une autre façon de pratiquer le voyage à l'intérieur d'un lieu continu? Est-ce simplement une manière de vendre différemment ce qui était déjà présenté autrement? À bien y penser, depuis la voie Appienne, construite au IVe siècle avant notre ère, les routes peuvent porter des noms différents de la destination qu'elles rejoignent. Oui, la route possède un nom, doit-on pour autant savoir pourquoi?

Martin Drouin est coordonnateur de l'Institut du patrimoine de l'Université du Québec à Montréal.

\section{Notes}

1 Le lieu historique national est géré, en collaboration avec Parcs Canada, par la Société Promotion Grand-Pré (consulter le site de la Société de promotion de Grand-Pré).

2 On peut consulter les onze routes de la province sur le site Internet officiel de l'Office de tourisme, tout comme les cinq routes créées au Nouveau-Brunswick qui ceinturent et coupent la province. L'Île-du-PrinceÉdouard offre quatre circuits qui sillonnent l'ensemble de l'île; la géographie a guidé le choix des circuits, sauf celui d'Anne, lié à l'héroïne du roman de Lucy Maud Montgomery (consulter le site officiel du tourisme de la Nouvelle-Écosse, le site touristique officiel du Nouveau-Brunswick et le site touristique de l'Île-du-Prince-Édouard).

3 Consulter la rubrique "La signalisation des routes et des circuits touristiques » (Tourisme Québec).

4 L'Institut européen des itinéraires culturels était créé en 1998 par le Conseil de l'Europe.

5 Les objectifs étaient de « promouvoir l'identification, l'étude et la mise en valeur des routes ou des itinéraires culturels et la signification qu'impliquent comme un ensemble dans lequel se trouve sa principale valeur, ainsi que la protection, l'entretien et la conservation de ses monuments, ensembles de bâtiments, restes archéologiques, paysages culturels et sites, tous en rapport entre eux à travers des valeurs culturelles et liens historiques ".

6 La "route de la soie ", la "route des esclaves", la "route du fer en Afrique » et la " route d'al-Andalus » figurent par les exemples expérimentés (UNESCO, 2002-2006).

\section{Bibliographie}

Bertrand, Jean-René (2005), "Saint-Jacques-de-Compostelle. Le patrimoine religieux, du contexte au prétexte", Téoros, vol. 24, n² 2, été, p. 26-38.

Bouchard, René (1981) Itinéraire toponymique du chemin du Roy : QuébecMontréal, Québec, Commission de toponymie, 1981, 89 p.

Circuit de découverte des chemins de Craig et Gosford (2006), [http://www. craig-gosford.ca/index.htm], (consulté le 4 juillet 2006).
Comité international des itinéraires culturels (1994), «Statuts», [www.ico mos-ciic.org], (consulté le 4 juillet 2006).

Comité international des itinéraires culturels (1998), "Antécédents et principes ", [www.icomos-ciic.org], (consulté le 4 juillet 2006).

Connexion-Lanaudière (2003), "Le chemin du Roy ", [http://collections.ic. gc.ca/cheminduroy/\#], (consulté le 4 juillet 2006).

Continuité (2003), numéro spécial : «Le patrimoine en circuits ", n 97, été 2003, p. 21-49.

De Blois Martin, Charles (1997), L'évolution des rapports entre les politiques du patrimoine et du tourisme au Québec, mémoire de maîtrise en sciences politiques, Université Laval, 112 p.

Gagnon, Serge (2003), L'échiquier touristique québécois, Sainte-Foy, Presses de l'Université du Québec, 359 p.

Historic Roads (2004), [www.historicroads.org], (consulté le 4 juillet 2006).

ICOMOS (1998), « Les chemins de Saint-Jacques-de-Compostelle en France", Patrimoine mondial, [http://whc.unesco.org/archive/advisory_ body_evaluation/868.pdf], (consulté le 2 juillet 2006).

Institut européen des itinéraires culturels (2006), [www.culture-routes.lu], (consulté le 4 juillet 2006).

Krestev, Todor (2003), « Itinéraires culturels - Synthèse des valeurs matérielles et immatérielle, le cas de l'Europe du Sud-Est ", La mémoire des lieux - Préserver le sens et les valeurs immatérielles des monuments et des sites, $14^{e}$ Assemblée générale et symposium scientifique de l'ICOMOS, [http://www.international.icomos.org/victoriafalls2003/papers_fre.htm], (consulté le 4 juillet).

MacLean, Terry (1995), Louisbourg Heritage: From Ruins to Reconstruction, Sydney (Nouvelle-Écosse), University College of Cape Breton Press, 170 p.

Site touristique de l'île-du-Prince-Édouard (2006), [http://www.gov.pe.ca/ visitorsguide/index. php3?number=1009647\&lang=F\#], (consulté le 4 juillet 2006).

Site officiel du tourisme de la Nouvelle-Écosse (2006), [www.nouvelle-ecos se.com], (consulté le 4 juillet 2006).

Site touristique officiel du Nouveau-Brunswick (2006), [www.tourismnew brunswick.ca], (consulté le 4 juillet 2006).

Société de promotion de Grand-Pré (2001), [www.grand-pre.com], (consulté le 4 juillet 2006).

Téoros (2003), numéro spécial : “Les routes à thèmes ", vol. 22, n² 2, été, p. 3-51.

Tourisme Québec (1995-2006), «La signalisation des routes et des circuits touristiques ", [www.bonjourquebec.com/mto/programmes/signali sation/circuits.html], [consulté le 4 juillet 2006).

Tourisme Yukon (2006), « La piste Chilkoot », [http://www.tourismeyukon.com/ Chilkoot/chilk_piste.htm], (consulté le 4 juillet 2006).

UNESCO (2002-2006), «Routes thématiques et tourisme culturel », [http:// portal.unesco.org/culture/fr/ev.php-URL_ID=4978\&URL_DO=DO_TOPIC \&URL_SECTION=201.html], (consulté le 4 juillet 2006). 